\title{
The Role of LRRK2 in Cell Signalling
}

Kirsten Harvey ${ }^{1 *}$, Tiago F. Outeiro ${ }^{2,3,4}$

Affiliation:

${ }^{1}$ Department of Pharmacology, UCL School of Pharmacy, University College London, 29-39 Brunswick Square, London WC1N 1AX, UK.

${ }^{2}$ Department of Experimental Neurodegeneration, Center for Nanoscale Microscopy and Molecular Physiology of the Brain, Center for Biostructural Imaging of Neurodegeneration, University Medical Center Göttingen, Göttingen, Germany

${ }^{3}$ Max Planck Institute for Experimental Medicine, Göttingen, Germany

${ }^{4}$ Institute of Neuroscience, The Medical School, Newcastle University, Framlington Place, Newcastle Upon Tyne, NE2 4HH, UK

*Correspondence: kirsten.harvey@ucl.ac.uk

Department of Pharmacology, UCL School of Pharmacy, University College London, 29-39

Brunswick Square, London WC1N 1AX, UK

\begin{abstract}
Parkinson's disease (PD) is a common late-onset neurodegenerative disorder known primarily for its motor features. Mutations and risk variants in LRRK2 cause familial and idiopathic forms of PD. Mutations segregating with disease are found in the LRRK2 GTPase and kinase domains, affecting catalytic activity and protein-protein interactions. This likely results in an overall gain of LRRK2 cell signalling function contributing to PD pathogenesis. This concept supports the development of LRRK2 kinase inhibitors as disease modifying treatments, at least for a subset of patients. However, LRRK2 function as a cell signalling protein with two catalytic and several protein-protein interaction domains is highly complex. For example, LRRK2 plays important roles in several inflammatory diseases, raising the possibility that it may mediate immune responses in PD. Consistently, LRRK2 mediated cell signalling was not only shown to be important for neuronal function, including neuronal development and homeostasis, but also for peripheral and central immune responses. The catalytic activity of LRRK2 is regulated by autophosphorylation, protein monomer/dimer cycling, and upstream kinases and GTPases, affecting its subcellular localisation and downstream signalling. Part of LRRK2-mediated signalling is likely facilitated by Rab-protein phosphorylation, affecting primarily membrane trafficking, including vesicle release at the trans-Golgi network. However, LRRK2 also displays intrinsic GTPase activity and functions as a signalling scaffold. As an example, LRRK2 was suggested to be part of the NRON complex and ß-catenin destruction complex, inhibiting NFAT and canonical Wnt signalling, respectively. In summary, continuous research into LRRK2 signalling function contributes to novel diagnostic and therapeutic concepts in PD.
\end{abstract}




\section{Introduction}

Parkinson's disease (PD) is a complex late-onset neurodegenerative disorder typically known for characteristic motor features [1]. In recent years, the contribution of gene mutations and genetic risk variants has been increasingly recognised as a cause of both familial and idiopathic forms of PD [1,2]. Almost 14 years ago, mutations in LRRK2 were identified as a cause of familial forms of PD [3,4]. Since then, genome wide association studies (GWAS) have also yielded an increasing amount of evidence supporting a major contribution of LRRK2 mutations and pathogenic risk variants to idiopathic PD [2]. Importantly, protective $L R R K 2$ variants were also identified and, some of them, were further characterised in cell biological and molecular assays, confirming the relevance of LRRK2 in modifying PD risk [5,6]. Interestingly, LRRK2 has not just been shown to play a role in PD but also to modify cancer risk, and to play a role in inflammatory diseases including infectious and autoimmune diseases $[7,8,9]$. This suggests a major role of LRRK2 in immune responses and raises the possibility that PD-associated LRRK2 mutants may also contribute neurodegeneration through effects on peripheral and/or central immune reactions.

Genetic and cell biological studies indicate that LRRK2 pathogenicity is conferred by a gain of function, likely partly through an increase of LRRK2 kinase activity [10,11]. This prompted the development of LRRK2 kinase inhibitors for clinical use as disease modifying agents [12]. However, the structure and function of LRRK2 as a cell signalling protein with two catalytic domains and several protein-protein interaction domains is highly complex. The regulation of LRRK2-mediated signalling is conferred by autophosphorylation, LRRK2 protein monomer/dimer cycling, upstream kinases and GTPases, downstream phosphorylation substrates, GTPase effectors, and participation in signalling complexes [13,14,15,16,17]. LRRK2 catalytic activity, autophosphorylation, heterophosphorylation and protein interactions determine the subcellular protein localisation [13,14,15,16,17]. Likewise, LRRK2 catalytic activity is also dependent on subcellular localisation and kinase activity, which was reported to be higher at membranes. LRRK2 PD-segregating mutations are all found in the catalytic RocCOR and kinase domains, and were shown to affect GTPase activity, kinase activity and/or protein-protein interactions [12,13,18,19, 20,21,22].

LRRK2 shows intrinsic kinase and GTPase activity but the LRRK2 interactome also suggests a manifold of interactions in diverse protein complexes [23]. This supports the idea that, owing at least partly to its large size, LRRK2 plays a particularly important role, via protein-protein interactions, as a signalling scaffold in several signalling pathways. LRRK2 scaffold function was suggested as part of the NRON (non-coding RNA repressor of NFAT) RNA protein complex, inhibiting NFAT signalling, and as part of the ß-catenin destruction complex, inhibiting canonical Wnt signalling [18,24]. LRRK2 also associates with the microtubule (MT) cytoskeleton and GSK3 $\beta$, thereby facilitating tau phosphorylation by GSK3 $\beta[19,21,25]$. In addition, LRRK2 was shown to participate in complex protein-protein interactions at the transGolgi network (TGN), with particular importance to local vesicle release $[16,17,26]$.

LRRK2-mediated cell signalling is important for neuronal function, neuronal development, homeostasis and synaptic function, but also plays a role in peripheral and central immune responses. Part of this function is likely facilitated by LRRK2-mediated Rab protein phosphorylation, with effects on membrane trafficking in endo- and exocytotic processes, including synaptic vesicle recycling $[9,10,12,13,15,23]$. This review aims to provide a conceptual view of LRRK2-associated cell signalling with a particular focus on the role of LRRK2 as a signalling scaffold. 


\section{LRRK2 catalytic activity}

LRRK2 belongs to the Roco proteins defined by a RocCOR (Roc: ras of complex proteins, COR: C-terminal of roc) tandem domain with intrinsic GTPase activity [27,28,29]. The RocCOR domain is followed by a serine/threonine specific kinase domain and a WD40 domain. At the N-terminus, armadillo (ARM), ankyrin (ANK) and leucine rich repeats (LRR) precede the LRRK2 GTPase domain (Fig. 1) [27,28,29]. ARM, ANK, LRR and WD40 domains are typical protein-protein interaction domains. However, important protein interactions were also described to take place with amino acids in-between the ANK and LRR repeats, and with the LRRK2 RocCOR tandem domain, including the interaction with 14-3-3 proteins and tubulins, respectively [28,29,30,31].

LRRK2 kinase activity is regulated by autophosphorylation of three residues in the kinase activation loop (Fig. 1) [32,33]. Further autophosphorylation sites are clustered in the Roc domain including the phosphosites T1410 and T1503 confirmed in cells $[12,13,28]$. Autophosphorylation of the Roc domain in turn effects kinase activity in a complex manner [34]. The in vivo confirmed S1292 autophosphorylation site in the LRR domain is dephosphorylated by the phosphatases PP1 and PP2. Importantly, most of the pathogenic LRRK2 mutants show increased autophosphorylation at S1292, but decreased phosphorylation at heterophosphorylation sites, in particular S935, whereas kinase inhibition leads to dephosphorylation of auto- and heterophosphorylation sites (Fig. 1) [12,13,28]. The complex effects and possible clinical pitfalls of LRRK2 inhibition on wild type and mutant LRRK2 phosphorylation states were recently reviewed [13].

Independently confirmed LRRK2 substrates are predominantly Rab proteins with a current focus on Rab8a, Rab10 and Rab29. Phosphorylation of Rabs by LRRK2 affects the membrane association of the Rab proteins. Therefore, LRRK2 mutations that increase its kinase activity lead to an accumulation of inactive Rab proteins at the membrane [15,35,36]. LRRK2 GTPase activity is conferred by the RocCOR tandem domain. The Roc domain shows close structural similarity to Ras proteins, and the COR domain facilitates LRRK2 dimerization, that was suggested to be important for LRRK2 GTPase activity [5,14,27,28,29,3]. Previous studies favoured the idea that LRRK2 belongs to a group of GTPases activated by dimerization [37]. However, recent work in bacterial Roco proteins homologous to LRRK2 suggests that Roco proteins are monomeric when GTP-bound, and predominantly dimeric in the GDP-bound and nucleotide-free states [14]. The authors propose a model in which GTP hydrolysis leads to protein dimerization of GDP-bound and/or nucleotide free Roco protein. The monomer-dimer cycle is then completed by GDP-GTP exchange facilitated by the low nucleotide affinity of Roco proteins, and the comparatively high physiological cellular GTP concentrations. In addition, the authors showed that introduction of an analogous LRRK2 PD mutant leads to dimer stabilization and a decrease in GTPase activity [14]. Despite the importance of these findings, it should be noted that LRRK2 contains additional dimerization domains in comparison to the bacterial Roco proteins investigated. This might further complicate catalytic activity regulation and the monomer dimer cycle likely affecting the balance between preferentially monomeric cytoplasmic and dimeric membrane bound LRRK2. Further considerations affecting the function of isolated LRRK2 domains and full length human LRRK2 have been reviewed previously by us and others [37,38]. The interaction between LRRK2 and Rab29 at the TGN, further described below, is a good example illustrating the complexity and interdependence of protein interactions, catalytic activity, and membrane association strength. 
Nucleotide binding to the RocCOR domain is also important for LRRK2 kinase function. Nonetheless, the exact mechanisms involved are unclear and not all results are easily reconciled, as reviewed by others [40]. In contrast, consistent results show that dimeric membrane-bound LRRK2 has increased kinase activity in comparison to monomeric cytosolic LRRK2, leaving the question of whether LRRK2 is predominantly GTP or GDP bound at membranes. In addition, autophosphorylation of residues in the Roc domain might affect protein-protein interactions, possibly influencing subcellular localisation of LRRK2 $[12,13,14,15,19,22,29,30,31,32,33,35,36]$. Importantly, all PD segregating mutations in the RocCOR tandem domain increase GTP-binding, decrease intrinsic GTPase activity, and increase Rab phosphorylation in cells, but not intrinsic kinase activity in kinase assays $[15,35,37]$. The LRRK2 G2019S mutation in the kinase domain increases intrinsic kinase activity but different reports are available on Rab phosphorylation in cells, whereas the I2020T kinase mutant increases Rab phosphorylation in cells but not intrinsic kinase activity in kinase assays [35,42]. However, Rab10 hyperphosphorylation was shown in G2019S knock-in mouse models, and a trend was also observed in G2019S PD-patient derived neutrophils $[35,39,41,42]$.

\section{LRRK2 mediated signalling}

Suggested LRRK2-mediated signalling pathways include growth factor and survival, as well as death receptor and Wnt signalling pathways [43,44,45]. Prominent examples are MAPK, Rac/PAK, Akt, PKA and Wnt pathways [20,43,46,47,48,49,50,51,48]. $\mathrm{Ca}^{2+}$-mediated pathways with relevance for innate immune function also deserve a separate mention, including pathways mediated by the receptor TLR, and the transcription factors NFAT (Nuclear factor of activated T-cells) and NFKB $[9,53,54,55]$. To date, upstream regulation of LRRK2 is best described for the heterophosphorylation by the constitutively active kinases GSK3 $\beta$ and CK1 $\alpha$. These kinases, as well as PKA, phosphorylate residues in-between the ANK and LRR repeats, whereas the phosphatase PP1 mediates dephosphorylation of these phosphosites, thereby affecting the interaction with 14-3-3 proteins. In turn, this affects the subcellular localisation of LRRK2, possibly because the LRRK2-14-3-3 interaction facilitates cytoplasmic localisation (Fig.2) [12,13,22,29,30,31,32,33].

The most established LRRK2 kinase substrates are the Rab proteins $[15,16,17,35,36,39,41]$. Evidence suggests that GTP-bound LRRK2 phosphorylates GTP-bound Rab proteins at membranes. This effect is likely increased by LRRK2 mutants, in particularly RocCOR mutants conferring increased GTP binding. In addition, Rab29 was described as an upstream GTPase of LRRK2 recruiting GTP-bound LRRK2 to the TGN, thereby enhancing LRRK2 autophosphorylation at S1292, and possibly increasing LRRK2 heterophosphorylation and simultaneously stimulating clearance of Golgi-derived vesicles $[16,17,26]$. This suggests a signalling loop in which LRRK2 is downstream and upstream of Rab proteins, in particular downstream of Rab29 and upstream of Rab8a, Rab10 and Rab29, respectively (Fig.2). In accordance with the idea of LRRK2 acting as a signalling scaffold, this complex interaction at the TGN might well occur inside a multi protein signalling complex. However, the role of LRRK2 as a signalling scaffold is possibly better described in the examples reported in the next section.

\section{LRRK2 as a signalling scaffold}

Signalling scaffolds typically interact with a multitude of proteins through protein-protein interaction domains, thereby bringing components of a signalling pathway in close proximity. Despite the above described catalytic activity of LRRK2, and likely relevance of kinase and GTPase activities for LRRK2 function, LRRK2 kinase activity is relatively low, and GTPase 
effectors have not been identified reproducibly, except for the LRRK2 kinase domain. The scale of described LRRK2 interacting proteins and proposed LRRK2 mediated signalling pathways also suggests that LRRK2 functions predominantly as a signalling scaffold $[13,15,23,29,43,56]$. In some instances, LRRK2 might function solely by strengthening a defined protein complex to facilitate, for e.g., phosphorylation by additional kinases (scenarios 4.1 and 4.2, described below). In other cases, evidence supports the additional relevance of LRRK2 catalytic activity (scenario 4.3). In the following sections, the possible scenarios will be considered using three distinct examples.

\subsection{LRRK2 scaffolding function in NFAT signalling}

Similar to LRRK2 signalling, NFAT signalling has particular importance in innate immune responses but also plays a role during neuronal development [24,57]. LRRK2 was first linked to NFAT signalling by the observation that LRRK2 deletion in mice increases NFAT signalling of resident immune cells in a model for Crohn's disease. LRRK2 knockout mice showed more severe symptoms of inflammatory bowel disease in comparison to wild type animals. This included rapid weight loss, severe diarrhea, bloody stools, extensive inflammatory infiltrates and mucosal damage and an increase in inflammatory cytokines such as IL-12 and IL-6. Similar underlying mechanisms have also been recently proposed in chronic rhinosinusitis and Aspergillus infections [24,58,59]. In these immune reactions, LRRK2 was suggested to function as a scaffold regulating NFAT signalling by a kinase-independent mechanism. LRRK2 was shown to interact with multiple protein and RNA components as part of the NRON complex, thereby stabilising this complex and enabling phosphorylation of NFAT by GSK3ß, CK1, and DYRK1A (Fig.3) [24]. Under these conditions NFAT phosphorylation retains NFAT in the cytoplasm. Increase of intracellular $\mathrm{Ca}^{2+}$, via several possible signalling pathways through extracellular receptor activation, with particular relevance in cells of the immune system, allow for calcineurin-mediated dephosphorylation of NFAT, nuclear localisation, and transcriptional activation of NFAT regulated genes such as IL-6. In this case, LRRK2 kinase activity does not seem to be relevant for the LRRK2 mediated NFAT signalling. Nonetheless, LRRK2 might also play an additional role in NFAT signalling through the interaction with dishevelled (DVL) proteins, important key regulators in all Wnt signalling pathways including the Wnt-Ca ${ }^{2+}$ pathway (Fig.3). Interestingly, canonical Wnt signalling has also been shown to be critical for the differentiation and survival of mature $T$ lymphocytes $[60,61,62]$. Further supporting the idea of crosstalk between NFAT and Wnt signalling in immune cells is a $\mathrm{Ca}^{2+}$ dependent direct interaction between NFAT and DVL1-3, inhibiting canonical Wnt signalling activity [63].

\subsection{LRRK2 scaffolding function at microtubules}

LRRK2 was shown to affect the MT and actin cytoskeleton, likely contributing to the observed effects of LRRK2 on axonal outgrowth and MT stability [5,19,49,64,65,66]. Effects on the actin cytoskeleton are partly conferred by the interaction of LRRK2 with small GTPases, GAPs and GEFs, such as rac1, rhoA, cdc42, ARFGAP1 and ARHGEF7. The LRRK2 Roc domain was shown to interact with MTs via a direct interaction with a selection of ß-tubulins. In accordance with this, LRRK2 deletion or LRRK2 mutants were shown to affect tubulin content and tubulin acetylation in diverse in vivo and in vitro systems [5,19,23,49,64,65,66,67]. Importantly, this does not exclude an interaction between several LRRK2 domains and other MT-associated components. Further regulation of the MT cytoskeleton might be conferred by the interaction between LRRK2 and GSK3ß, a well-known tau kinase [18,21,25]. Most importantly, LRRK2 was shown to affect tau phosphorylation at MTs, most likely through the interaction with GSK3ß and MTs, thereby increasing tau phosphorylation and affecting MT stability. Although LRRK2 and tau were shown to interact [68] a direct effect of LRRK2 on 
tau phosphorylation in still under debate. Most evidence points to a scaffolding role of LRRK2 that facilitates tau phosphorylation at MTs by a mechanism independent of LRRK2 kinase activity. Importantly, the interaction strength between LRRK2 and GSK3ß was shown to be affected by the G2019S mutation, allowing for a mechanism of inducing axonal degeneration during the pathogenesis of PD (Fig.1,3) [18,21,25,69,70,71]. In addition, it is important to consider that LRRK2 might also affect tau phosphorylation by GSK3ß at MTs through several different pathways, likely including a Wnt signalling pathway [20,73]. A similar mechanism of increased tau phosphorylation through an interaction between LRRK2 and cdk5 at MTs has been suggested and shown to be affected by the R1628P mutation $[69,72]$.

\subsection{LRRK2 scaffolding function in Wnt signalling pathways}

Wnt signalling pathways regulate diverse cell biological functions in the CNS and peripheral organs by affecting transcriptional activity and cytoskeletal stability, overall supporting cell division, growth and homeostasis. LRRK2 might potentially affect all three Wnt signalling branches through the interaction with DVL proteins. DVL functions as a central cytosolic key regulator in the canonical ß-catenin mediated, and the non-canonical $\mathrm{PCP}$ and $\mathrm{Ca}^{2+} \mathrm{Wnt}$ signalling pathways $[11,18,20,52,73]$. Additional LRRK2 interactions were shown with multiple components of the ß-catenin destruction complex, including GSK3ß, which is relevant for canonical Wnt signalling under basal conditions and the canonical Wnt Lrp6 co-receptor relevant under activated signalling conditions. LRRK2 interacting Wnt-PCP pathway components include the cytoplasmic PRICKLE1 and the Vangl2 receptor (Fig.4) $[11,18,20,21,25,52,73]$. The importance of LRRK2 in Wnt-PCP signalling was shown by LRRK2 overexpression in Xenopus embryos, leading to alterations of embryonic convergenceextension movements [48].

LRRK2-mediated Wnt signalling is highly dependent on pathway activation. LRRK2 was shown to be a suppressor of canonical Wnt signalling under basal condition as part of the Bcatenin destruction complex. In the B-catenin destruction complex LRRK2 most likely plays a role as a signalling scaffold facilitating phosphorylation of B-catenin by CK1 and GSK3ß resulting in the degradation of phosphorylated $\beta$-catenin. However, upon pathway activation for example with the canonical Wnt ligand Wnt3a or DVL protein overexpression LRRK2 was shown to increase canonical Wnt signalling activity $[11,18,45,52,73]$.

Under most conditions, the canonical Wnt signalling and the non-canonical Wnt-PCP pathways are antagonistic to each other [74,75]. Interestingly, LRRK2 was identified as a possible switch between these two related pathways by changing interactions with canonical and non-canonical Wnt signalling components [52]. As described above, LRRK2 suppresses canonical Wnt signalling while interacting with proteins within the ß-catenin destruction complex but becomes an activator of this pathway upon canonical Wnt signalling activation, most likely through an interaction with DVL proteins. However, the LRRK2-DVL interaction can be affected by adding a third non-canonical Wnt signalling protein, PRICKLE1. The LRRK2PRICKLE1 interaction activates the non-canonical PCP pathway and simultaneously suppresses the canonical Wnt signalling pathway $[11,18,45,52,73]$. This switch between these two pathways might rely on relatively small changes of protein levels at different subcellular localisations dependent on activation states of Wnt signalling pathway branches.

Even though the role of LRRK2 in Wnt signalling seems to be predominantly as a signalling scaffold, it was shown that inhibition of LRRK2 kinase activity decreases canonical Wnt signalling in wild type LRRK2 cell models under activating conditions $[11,18]$. This might be through an effect on LRRK2 autophosphorylation, thereby affecting protein-protein 
interactions, as described above. Even though the precise mechanism is unknown, it is important to note that all PD-segregating LRRK2 mutants and risk variants decrease canonical Wnt signalling under basal and activating conditions, whereas a protective variant increases canonical Wnt signalling. This shows a clear negative correlation between LRRK2-mediated PD risk and canonical Wnt signalling activity [5,11,18,36,45,51]. Therefore, activation of canonical Wnt signalling in fine boundaries emerges as an attractive therapeutic target.

\section{Perspectives}

The investigation of LRRK2 is highly relevant in the context of PD for three main reasons: (i) LRRK2 mutations and risk variants contribute substantially to PD risk; (ii) the clinical presentation of LRRK2 patients is indistinguishable from that of idiopathic patients; and (iii) as a signalling protein, LRRK2 lends itself well to regulation by pharmacological therapeutic intervention, currently mostly realised through LRRK2 kinase inhibition $[1,2,12]$.

The role of LRRK2 in signalling pathways is well-established. The multitude of protein-protein interactions, the relatively low catalytic activity, the very few confirmed kinase substrates and GTPase effectors, suggest that LRRK2 mainly functions as a signalling scaffold. LRRK2 likely plays roles in the fine regulation of several signalling pathways with several cell biological functions. LRRK2 mutants often confer very subtle gain of function effects on these physiological cell pathways likely exacerbated by aging, environmental toxins and, possibly, triggers of immune and/or autoimmune reactions.

Despite the tremendous progress in LRRK2 research over the last decade, the detailed mechanisms of physiological and pathophysiological LRRK2 function needs further investigation. The interdependence and fine regulation of LRRK2-mediated cell signalling, especially in different neuronal and immune cell populations, is a major focus of current studies. This is also highly relevant in PD and in other LRRK2-related diseases, and is likely to continue in the future. The importance of LRRK2 as a therapeutic target for PD is undisputed, but concerns regarding the safety of LRRK2 kinase inhibition encourages the search for agents that can regulate other LRRK2 domains or pathway components, with the ultimate aim of finding a disease-modifying therapy for PD targeting LRRK2-mediated cell signalling pathways. 


\section{References}

1. Shulman, J.M., De Jager, P.L. \& Feany, M.B. (2011) Parkinson's disease: genetics and

Pathogenesis. Annu. Rev. Pathol. 6, 193-222.

2. Chang, D., Nalls, M.A., Hallgrímsdóttir, I.B., Hunkapiller, J., van der Brug, M., Cai, F. et al. (2017) A meta-analysis of genome-wide association studies identifies 17 new Parkinson's disease risk loci. Nat. Genet. 49, 1511-1516.

3. Paisán-Ruíz, C., Jain, S., Evans, E.W., Gilks, W.P., Simón, J., van der Brug, M. et al. (2004). Cloning of the gene containing mutations that cause PARK8-linked Parkinson's disease. Neuron 44, 595-600.

4. Zimprich, A., Biskup, S., Leitner, P., Lichtner, P., Farrer, M., Lincoln, S. et al. (2004). Mutations in LRRK2 cause autosomal-dominant parkinsonism with pleomorphic pathology. Neuron 44, 601-607.

5. Nixon-Abell, J., Berwick, D. C., Granno, S., Spain, V. A., Blackstone, C. and Harvey, K. (2016) Protective LRRK2 R1398H Variant Enhances GTPase and Wnt Signaling Activity. Front. Mol. Neurosci. 9, 18

6. Hui, K.Y., Fernandez-Hernandez, H., Hu, J., Schaffner, A., Pankratz, N., Hsu, N.Y. et al. (2018)

Functional variants in the LRRK2 gene confer shared effects on risk for Crohn's disease and Parkinson's disease.Sci. Transl. Med. 10, eaai7795.

7. Tacik, P., Curry, S., Fujioka, S., Strongosky,, A., Uitti, R.J., van Gerpen, J.A. et al. (2016) Cancer in Parkinson's disease. Parkinsonism Relat Disord 31, 28-33

8. Agalliu, I., San Luciano, M., Mirelman, A., Giladi, N., Waro, B., Aasly, J. et al. (2015) Higher frequency of certain cancers in LRRK2 G2019S mutation carriers with Parkinson disease: a pooled analysis. JAMA Neurol 72, 58-65.

9. Dzamko, N.L. (2017) LRRK2 and the Immune System. Adv Neurobiol. 14, 123-143.

10. Islam, M.S., Moore, D.J. (2017) Mechanisms of LRRK2-dependent neurodegeneration: role of enzymatic activity and protein aggregation. Biochem Soc Trans. 45, 163-172.

11. Berwick, D.C., Javaheri, B., Wetzel, A., Hopkinson, M., Nixon-Abell, J., Grannò, S. et al. (2017) Pathogenic LRRK2 variants are gain-of-function mutations that enhance LRRK2mediated repression of $\beta$-catenin signaling. Mol Neurodegener. 12:9.

12. West, A.B. (2017) Achieving neuroprotection with LRRK2 kinase inhibitors in Parkinson disease. Exp Neurol. 298, 236-245.

13. De Wit, T., Baekelandt, V., and Lobbestael, E. (2018). LRRK2 Phosphorylation: Behind the Scenes. Neuroscientist. 24, 486-500. 
14. Deyaert, E., Wauters, L., Guaitoli, G., Konijnenberg, A., Leemans, M., Terheyden et al. (2017). A homologue of the Parkinson's diseaseassociated protein LRRK2 undergoes a monomer-dimer transition during GTP turnover. Nat. Commun. 8, 1-12.

15. Madero-Pérez, J., Fdez, E., Fernández, B., Lara Ordóñez, A.J., Blanca Ramírez, M., Romo Lozano, M. et al. (2017) Cellular effects mediated by pathogenic LRRK2: homing in on Rabmediated processes. Biochem. Soc. Trans. 8, 147-154.

16. Liu, Z., Bryant, N., Kumaran, R., Beilina, A., Abeliovich, A., Cookson, M.R. et al (2018) LRRK2 phosphorylates membrane-bound Rabs and is activated by GTP-bound Rab7L1 to promote recruitment to the trans-Golgi network. Hum. Mol. Genet. 27, 385-395.

17. Purlyte, E., Dhekne, H.S., Sarhan, A.R., Gomez, R., Lis, P., Wightman, M. et al. (2018) Rab29 activation of the Parkinson's disease-associated LRRK2 kinase. EMBO J. 37, 1-18.

18. Berwick, D.C., Harvey, K. (2012) LRRK2 functions as a Wnt signaling scaffold, bridging cytosolic proteins and membrane-localized LRP6. Hum. Mol. Genet. 21, 4966-79.

19. Law, B.M., Spain, V.A., Leinster, V.H., Chia, R., Beilina, A., Cho, H.J. et al (2014) A direct interaction between leucine-rich repeat kinase 2 and specific $\beta$-tubulin isoforms regulates tubulin acetylation. J. Biol. Chem. 289, 895-908.

20. Sancho, R.M., Law, B.M. and Harvey, K. (2009) Mutations in the LRRK2 Roc-COR tandem domain link Parkinson's disease to Wnt signalling pathways. Hum. Mol. Genet. 18, 3955-3968.

21. Lin, C.H., Tsai, P.I., Wu, R.M. and Chien, C.T. (2010) LRRK2 G2019S mutation induces dendrite degeneration through mislocalization and phosphorylation of tau by recruiting autoactivated GSK3ß. J. Neurosci. 30, 13138-13149.

22. Berger, Z., Smith, K.A., and LaVoie, M.J. (2010). Membrane localization of LRRK2 is associated with increased formation of the highly active LRRK2 dimer and changes in its phosphorylation. Biochemistry 49, 5511-5523.

23. Tomkins, J.E., Dihanich, S., Beilina, A., Ferrari, R., Ilacqua, N., Cookson, M.R. et al. (2018) Comparative Protein Interaction Network Analysis Identifies Shared and Distinct Functions for the Human ROCO Proteins. Proteomics. 18, e1700444.

24. Liu, Z., Lee, J., Krummey, S., Lu, W., Cai, H., Lenardo, M.J. (2011) The kinase LRRK2 is a regulator of the transcription factor NFAT that modulates the severity of inflammatory bowel disease. Nat. Immunol. 12, 1063-70.

25. Kawakami, F., Shimada, N., Ohta, E., Kagiya, G., Kawashima, R., Maekawa, T., Maruyama, H. et al. (2014) Leucine-rich repeat kinase 2 regulates tau phosphorylation through direct activation of glycogen synthase kinase-3ß. FEBS J. 281, 3-13.

26. Beilina, A., Rudenko, I.N., Kaganovich, A., Civiero, L., Chau, H., Kalia, S.K. et al. (2014) Unbiased screen for interactors of leucine-rich repeat kinase 2 supports a common pathway for sporadic and familial Parkinson disease. Proc. Natl. Acad. Sci. U.S.A. 111, 2626-2631. 
27. Bosgraaf, L., Van Haastert, P.J. (2003). Roc, a Ras/GTPase domain in complex proteins. Biochim. Biophys. Acta 1643, 5-10.

28. Mata, I.F., Wedemeyer, W.J., Farrer, M.J., Taylor, J.P., and Gallo, K.A. (2006). LRRK2 in Parkinson's disease: protein domains and functional insights. Trends Neurosci. 29, 286-293.

29. Mills, R.D., Mulhern, T.D., Liu, F., Culvenor, J.G., and Cheng, H.C. (2014). Prediction of the Repeat Domain Structures and Impact of Parkinsonism-Associated Variations on Structure and Function of all Functional Domains of Leucine-rich Repeat Kinase 2 (LRRK2). Hum. Mutat. 35, 395-412.

30. Nichols, R.J., Dzamko, N., Morrice, N.A., Campbell, D.G., Deak, M., Ordureau, A. et al. (2010). 14-3-3 binding to LRRK2 is disrupted by multiple Parkinson's disease-associated mutations and regulates cytoplasmic localization. Biochem. J. 430, 393-404.

31. Li, X., Wang, Q.J., Pan, N., Lee, S., Zhao, Y., Chait, B.T. et al. (2011). PhosphorylationDependent 14-3-3 Binding to LRRK2 Is Impaired by Common Mutations of Familial Parkinson's Disease. PLOS ONE 6, 1-13.

32. Greggio, E., Zambrano, I., Kaganovich, A., Beilina, A., Taymans, J.-M., Daniëls, V. et al. (2008). The Parkinson disease-associated leucine-rich repeat kinase 2 (LRRK2) is a dimer that undergoes intramolecular autophosphorylation. J. Biol. Chem. 283, 16906-16914.

33. Li, X., Moore, D.J., Xiong, Y., Dawson, T.M., and Dawson, V.L. (2010). Reevaluation of phosphorylation sites in the Parkinson disease-associated leucine-rich repeat kinase 2. J. Biol. Chem. 285, 29569-29576.

34. Athanasopoulos, P.S., Heumann, R., Kortholt, A. (2018) The role of (auto)phosphorylation in the complex activation mechanism of LRRK2. Biol. Chem. 399, 643-647.

35. Steger, M., Tonelli, F., Ito, G., Davies, P., Trost, M., Vetter, M. et al. (2016). Phosphoproteomics reveals that Parkinson's disease kinase LRRK2 regulates a subset of Rab GTPases. ELife 5, 1-28.

36. Steger, M., Diez, F., Dhekne, H.S., Lis, P., Nirujogi, R.S., Karayel, O. et al. (2017). Systematic proteomic analysis of LRRK2-mediated Rab GTPase phosphorylation establishes a connection to ciliogenesis. ELife 6, 1-22.

37. Nixon-Abell, J., Berwick, D.C., and Harvey, K. (2016). L'RRK de Triomphe: a solution for LRRK2 GTPase activity? Biochem. Soc. Trans. 44, 1625-1634.

38. Terheyden, S., Nederveen-Schippers, L.M., Kortholt, A. (2016) The unconventional Gprotein cycle of LRRK2 and Roco proteins. Biochem Soc Trans. 44, 1611-1616.

39. Fan Y, Howden AJM, Sarhan AR, Lis P, Ito G, Martinez TN et al. (2018) Interrogating Parkinson's disease LRRK2 kinase pathway activity by assessing Rab10 phosphorylation in human neutrophils. Biochem. J. 475, 23-44.

40. Gilsbach, B.K., Eckert, M., Gloeckner, C.J. (2018) Regulation of LRRK2: insights from structural and biochemical analysis. Biol. Chem. 399, 637-642. 
41. Ito, G., Katsemonova, K., Tonelli, F., Lis, P., Baptista, M.A.S., Shpiro, N. et al. (2016) Phos-tag analysis of Rab10 phosphorylation by LRRK2: a powerful assay for assessing kinase function and inhibitors. Biochem. J. 473, 2671-2685.

42. Lis, P., Burel, S., Steger, M., Mann, M., Brown, F., Diez, F. et al. (2018). Development of phospho-specific Rab protein antibodies to monitor in vivo activity of the LRRK2

Parkinson's disease kinase. Biochem. J. 475, 1-22.

43. Berwick, D.C., Harvey, K (2011) LRRK2 signaling pathways: the key to unlocking neurodegeneration? Trends Cell Biol. 21, 257-65.

44. Rideout, H.J., Re, D.B. (2017) LRRK2 and the "LRRKtosome" at the Crossroads of Programmed Cell Death: Clues from RIP Kinase Relatives. Adv. Neurobiol. 14, 193-208.

45. Berwick, D.C., Harvey, K. (2014) The regulation and deregulation of Wnt signaling by PARK genes in health and disease. J. Mol. Cell. Biol. 6: 3-12.

46. Chen, C.Y., Weng, Y.H., Chien, K.Y., Lin, K.J., Yeh, T.H., Cheng, Y.P. et al. (2012). (G2019S) LRRK2 activates MKK4-JNK pathway and causes degeneration of SN dopaminergic neurons in a transgenic mouse model of PD. Cell Death Differ. 19, 1623-1633

47. Civiero, L., Cirnaru, M.D., Beilina, A., Rodella, U., Russo, I., Belluzzi, E. et al. (2015) Leucine-rich repeat kinase 2 interacts with p21-activated kinase 6 to control neurite complexity in mammalian brain. J. Neurochem. 135, 1242-1256.

48. Chuang, C.-L., Lu, Y.-N., Wang, H.-C. and Chang, H.-Y. (2014) Genetic dissection reveals that Akt is the critical kinase downstream of LRRK2 to phosphorylate and inhibit FOXO1, and promotes neuron survival. Hum. Mol. Genet. 23, 5649-5658.

49. Greggio, E., Bubacco, L., Russo, I. (2017) Cross-talk between LRRK2 and PKA: implication for Parkinson's disease? Biochem. Soc. Trans. 45, 261-267.

50. Russo, I., Berti, G., Plotegher, N., Bernardo, G., Filograna, R., Bubacco, L. et al. (2015) Leucine-rich repeat kinase 2 positively regulates inflammation and down-regulates NF- $\mathrm{B}$ p50 signaling in cultured microglia cells. J. Neuroinflammation 12, 230.

51. Berwick, D.C., Javaheri, B., Wetzel, A., Hopkinson, M., Nixon-Abell, J., Grannò, S. et al. (2017) Pathogenic LRRK2 variants are gain-of-function mutations that enhance LRRK2mediated repression of $\beta$-catenin signaling. Mol. Neurodegener. 12:9.

52. Salašová, A., Yokota, C., Potěšil, D., Zdráhal, Z., Bryja, V., Arenas, E (2017) A proteomic analysis of LRRK2 binding partners reveals interactions with multiple signaling components of the WNT/PCP pathway. Mol. Neurodegener. 12:54.

53. Russo, I., Bubacco, L. and Greggio, E. (2014) LRRK2 and neuroinflammation: partners in crime in Parkinson's disease? J. Neuroinflammation 11:52 
54. Dzamko, N., Inesta-Vaquera, F., Zhang, J., Xie, C., Cai, H., Arthur, S. et al. (2012) The IkappaB kinase family phosphorylates the Parkinson's disease kinase LRRK2 at Ser935 and Ser910 during Toll-like receptor signaling. PLoS One 7:e39132

55. Lee, H., James, W.S., Cowley, S.A. (2017) LRRK2 in peripheral and central nervous system innate immunity: its link to Parkinson's disease. Biochem. Soc. Trans. 45, 131-139.

56. Jaleel, M., Nichols, R.J., Deak, M., Campbell, D.G., Gillardon, F., Knebel, A. et al. (2007) LRRK2 phosphorylates moesin at threonine-558: characterization of how Parkinson's disease mutants affect kinase activity. Biochem. J. 405, 307-317.

57. Moore, D.L., Goldberg, J.L. (2011) Multiple transcription factor families regulate axon growth and regeneration. Dev. Neurobiol. 71, 1186-1211.

58. Ma Y, Zheng C, Shi L. (2018) The kinase LRRK2 is differently expressed in chronic rhinosinusitis with and without nasal polyps. Clin. Transl. Allergy. 12;8:8.

59. Wong, A.Y.W., Oikonomou, V., Paolicelli, G., De Luca, A., Pariano, M., Fric, J. et al. (2018) Leucine-Rich Repeat Kinase 2 Controls the Ca2+/Nuclear Factor of Activated T Cells/IL-2 Pathway during Aspergillus Non-Canonical Autophagy in Dendritic Cells. Front. Immunol. 9:210.

60. Gattinoni, L., Ji, Y., Restifo, N,P. (2010) Wnt/beta-catenin signaling in T-cell immunity and cancer immunotherapy. Clin. Cancer Res. 16, 4695-701

61. Staal, F.J., Arens, R. (2016) Wnt Signaling as Master Regulator of T-Lymphocyte Responses: Implications for Transplant Therapy. Transplantation. 100, 2584-2592.

62. Xue, H.H., Zhao, D.M. (2012) Regulation of mature T cell responses by the Wnt signaling pathway. Ann. NY Acad. Sci. 1247, 16-33.

63. Huang, T., Xie, Z., Wang, J., Li, M., Jing, N., Li, L. (2011) Nuclear factor of activated T cells (NFAT) proteins repress canonical Wnt signaling via its interaction with Dishevelled (Dvl) protein and participate in regulating neural progenitor cell proliferation and differentiation. J. Biol. Chem. 286: 37399-37405.

64. Blanca Ramírez, M., Lara Ordóñez, A.J., Fdez, E., Hilfiker, S. (2017) LRRK2: from kinase to GTPase to microtubules and back. Biochem. Soc. Trans. 45, 141-146.

65. Pellegrini, L., Wetzel, A., Grannó, S., Heaton, G., Harvey, K. (2017) Back to the tubule: microtubule dynamics in Parkinson's disease. Cell. Mol. Life. Sci. 74, 409-434.

66. Godena, V.K., Brookes-Hocking, N., Moller, A., Shaw, G., Oswald, M., Sancho, R.M. et al. (2014) Increasing microtubule acetylation rescues axonal transport and locomotor deficits caused by LRRK2 Roc-COR domain mutations. Nat. Commun. 5:5245.

67. Pellegrini, L., Hauser, D.N., Li, Y., Mamais, A., Beilina, A., Kumaran, R. et al. (2018) Proteomic analysis reveals co-ordinated alterations in protein synthesis and degradation pathways in LRRK2 knockout mice. Hum. Mol. Genet. 27, 3257-3271. 
68. Guerreiro, P.S., Gerhardt, E., Lopes da Fonseca, T., Bähr, M., Outeiro, T.F., Eckermann, K. (2016) LRRK2 Promotes Tau Accumulation, Aggregation and Release. Mol. Neurobiol. 53, 3124-3135.

69. Shanley, M.R., Hawley, D., Leung, S., Zaidi, N.F., Dave, R., Schlosser, K.A. et al. (2015) LRRK2 Facilitates tau Phosphorylation through Strong Interaction with tau and cdk5. Biochemistry. 54: 5198-208.

70. Kawakami, F., Yabata, T., Ohta, E., Maekawa, T., Shimada, N., Suzuki, M. et al. (2012). LRRK2 phosphorylates tubulin-associated tau but not the free molecule: LRRK2-mediated regulation of the tau-tubulin association and neurite outgrowth. PLoS One 7, e30834.

71. Hamm, M., Bailey, R., Shaw, G., Yen, S.H., Lewis, J., Giasson, B.I. (2015) Physiologically relevant factors influence tau phosphorylation by leucine-rich repeat kinase 2. J. Neurosci. Res. 93, $1567-80$.

72. Shu, Y., Ming, J., Zhang, P., Wang, Q., Jiao, F., Tian, B. (2016) Parkinson-Related LRRK2 Mutation R1628P Enables Cdk5 Phosphorylation of LRRK2 and Upregulates Its Kinase Activity. PLoS One 11:e0149739.

73. Berwick, D.C., Harvey, K. (2012) The importance of Wnt signalling for neurodegeneration in Parkinson's disease. Biochem. Soc. Trans. 40, 1123-1128.

74. Niehrs C. The complex world of WNT receptor signalling. Nat Rev Mol Cell Biol. 2012;13:767-79.

75. Sato A, Yamamoto H, Sakane H, Koyama H, Kikuchi A. Wnt5a regulates distinct signalling pathways by binding to Frizzled2. EMBO J. 2010;29:41-54. 
Abbreviations:

ANK: ankyrin repeats

ARM: armadillo repeats

COR: C-terminal of roc

DVL: dishevelled

GAP: Guanine activating proteins

LRR: leucine rich repeats

MT: microtubules

NFAT: Nuclear factor of activated T-cells

NRON: Non-coding RNA repressor of NFAT

PD: Parkinson's disease

Roc: ras of complex proteins

TGN: trans-Golgi network

\section{Conflicts of interest}

The authors declare no conflict of interest.

\section{Funding information}

$\mathrm{KH}$ is supported in part by the Medical Research Council [MR/M00676X/1]. TFO is supported by the DFG Center for Nanoscale Microscopy and Molecular Physiology of the Brain (CNMPB) and by DFG SFB1286 (project B8). The funders had no role in the decision to publish, or preparation of the manuscript. 


\section{Figure Legends}

Fig 1 LRRK2 signalling regulation. Top: LRRK2 is a GTPase and kinase allowing for downstream regulation of GTPase effectors and kinase substrates. The kinase activity also confers autophosphorylation of phosphosites in the kinase activation loop, with S2032 and T2035 phosphorylation required for LRRK2 kinase activity, and the LRRK2 Roc and LRR domains, with likely importance for protein-protein interactions. The RocCOR domain has intrinsic GTPase activity and GTP binding is important for kinase activity. Middle: LRRK2 functions as a signalling scaffold bringing different components of a signalling pathway together. Bottom: An example of LRRK2 scaffold function. The LRRK2 Roc domain interacts with MTs through a direct interaction with specific ß-tubulins. LRRK2 also interacts with GSK3ß and increases phosphorylation of the GSK3ß Y216 phosphosite thereby increasing indirectly tau phosphorylation by GSK3ß at MTs.

Fig 2 Upstream regulation of LRRK2. CK1alpha, IKK, PKA and PP1 were shown to be upstream regulatory kinases and phosphatases of LRRK2 respectively affecting predominantly the phosphorylation of serine residues in-between the ANK and LRR repeats. This heterophosphorylation is required for the interaction with 14-3-3 proteins with importance for the subcellular localisation of LRRK2. It was further shown that GTP-bound LRRK2 recruitment to membranes by GTP-bound Rab proteins results in an increase of LRRK2 autophosphorylation. In particular, Rab29 functions as an upstream GTPase of LRRK2 recruiting LRRK2 to the TGN resulting in an increase in LRRK2 kinase activity. LRRK2Rab29 interaction also seems required for constitutive phosphorylation of serine residues inbetween the ANK and LRR repeats. Kinases and GTPases, and their corresponding phosphorylation sites and interactors are colour coded using light yellow, blue and lilac.

Fig 3 LRRK2-mediated NFAT signalling in innate myeloid cells. LRRK2 knockout was shown to increase NFAT signalling in a model of Crohn's disease. Here, LRRK2 functions as a scaffold as part of the NRON complex facilitating NFAT phosphorylation by CK1, GSK3ß and DYRK1A. Upstream signalling, possibly through TLR4, Dectin and/or Wnt/Ca ${ }^{2+}$ signalling, leads to an increase in intracellular $\mathrm{Ca}^{2+}$, activation of the calcineurin phosphatase, dephosphorylation and nuclear localisation of NFAT, allowing NFAT downstream gene transcription.

Fig 4 LRRK2-mediated Wnt signalling. LRRK2 was found to interact with several components of the canonical ß-catenin mediated (left) and non-canonical PCP (right) Wnt pathways. These two pathways are known to act mostly antagonistically. LRRK2 effects on the signalling pathways is highly dependent on the activation state of the pathways as well as interacting proteins. As part of the ß-catenin destruction complex LRRK2 interacts with GSK3ß, axin and ß-catenin allowing ß-catenin phosphorylation by CK1 and GSK3ß. This leads to the destruction of $\beta$-catenin in a proteasomal pathway. This suppressor function is further increased by LRRK2 PD mutations in line with a gain of LRRK2 function. The canonical Wnt signalling pathway is activated upon binding of canonical Wnt ligands such as Wnt3a to Frizzled receptors leading to the recruitment of the LRRK2 interacting DVL proteins and other components of the ß-catenin destruction complex to the cell membrane. Here LRRK2 interacts in addition with the Lrp6 canonical Wnt co-receptor and DVL proteins interact with Frizzled receptors. Under activating conditions LRRK2 functions as a canonical Wnt signalling enhancer. However, when LRRK2 is bound to the PCP pathway component PRICKLE1 it decreases canonical Wnt signalling and increases PCP signalling activity. 
Figure 1
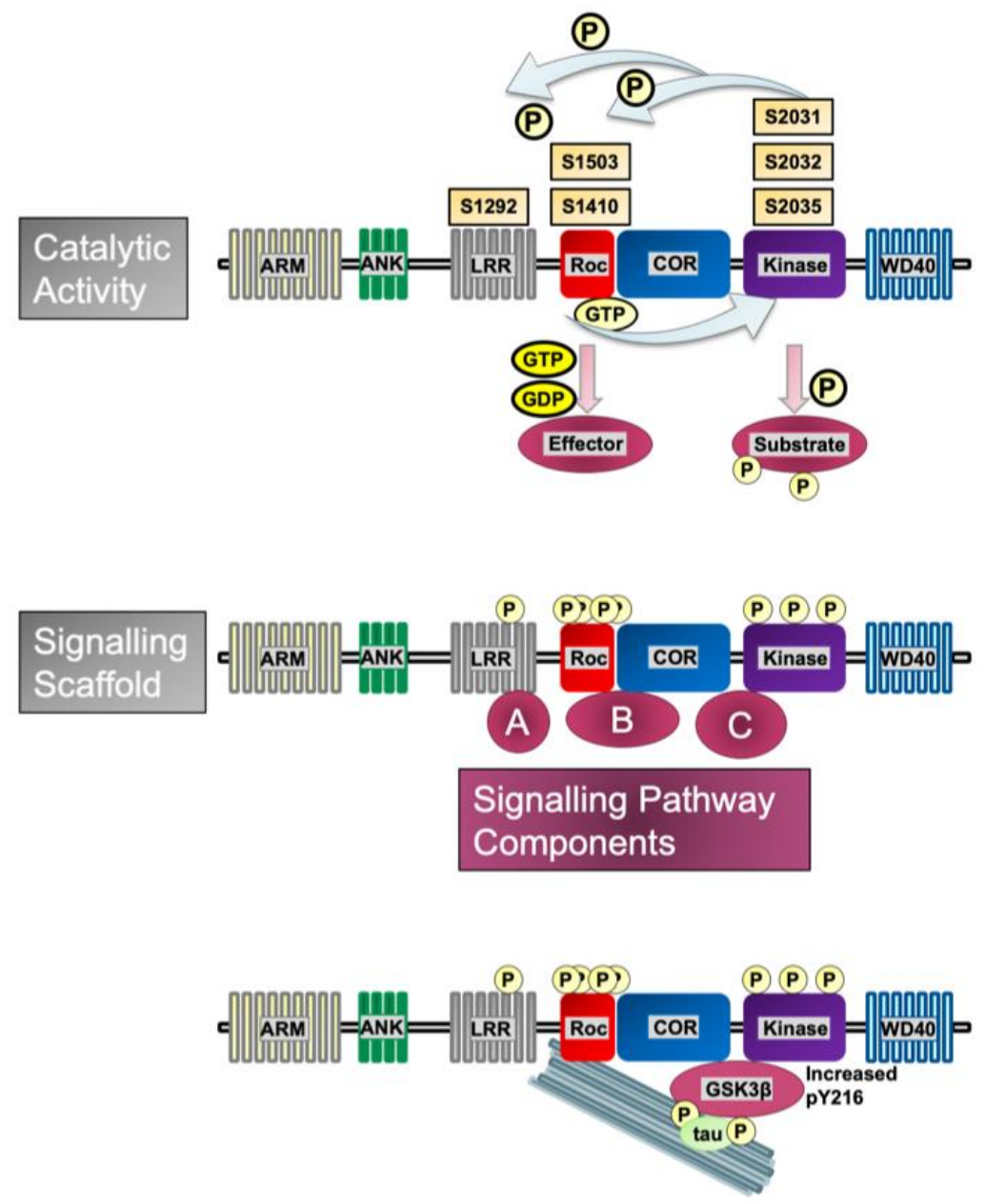

Fig 1 LRRK2 signalling regulation. Top: LRRK2 is a GTPase and kinase allowing for downstream regulation of GTPase effectors and kinase substrates. The kinase activity also confers autophosphorylation of phosphosites in the kinase activation loop, with S2032 and T2035 phosphorylation required for LRRK2 kinase activity, and the LRRK2 Roc and LRR domains, with likely importance for protein-protein interactions. The RocCOR domain has intrinsic GTPase activity and GTP binding is important for kinase activity. Middle: LRRK2 functions as a signalling scaffold bringing different components of a signalling pathway together. Bottom: An example of LRRK2 scaffold function. The LRRK2 Roc domain interacts with MTs through a direct interaction with specific ß-tubulins. LRRK2 also interacts with GSK3ß and increases phosphorylation of the GSK3ß Y216 phosphosite thereby increasing indirectly tau phosphorylation by GSK3ß at MTs. 
Figure 2

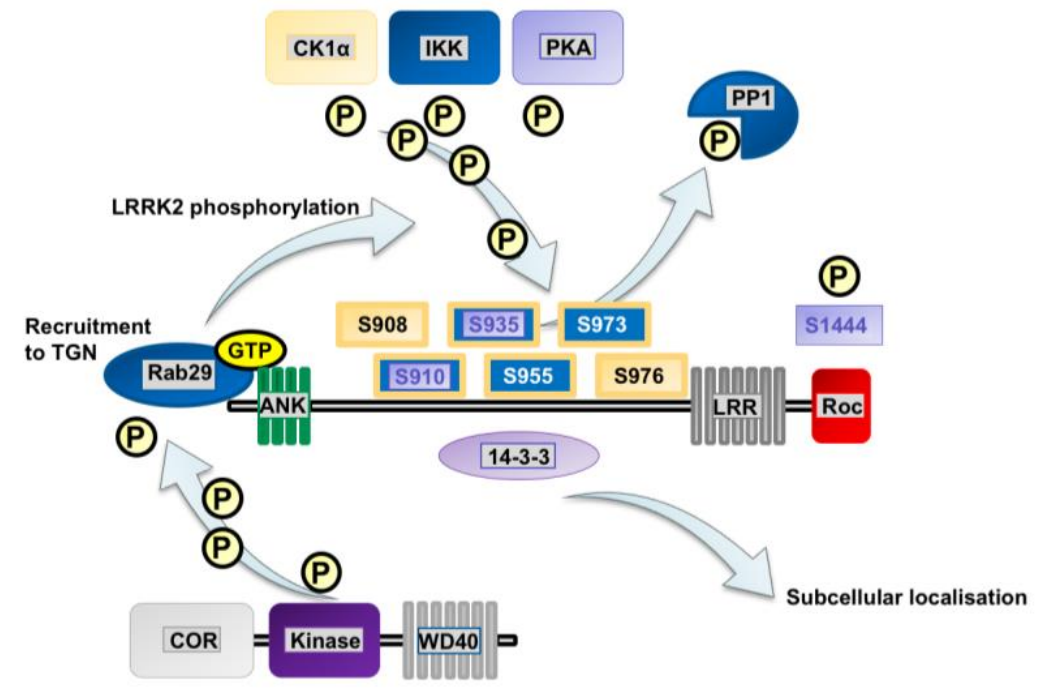

Fig 2 Upstream regulation of LRRK2. CK1alpha, IKK, PKA and PP1 were shown to be upstream regulatory kinases and phosphatases of LRRK2 respectively affecting predominantly the phosphorylation of serine residues in-between the ANK and LRR repeats. This heterophosphorylation is required for the interaction with 14-3-3 proteins with importance for the subcellular localisation of LRRK2. It was further shown that GTP-bound LRRK2 recruitment to membranes by GTP-bound Rab proteins results in an increase of LRRK2 autophosphorylation. In particular, Rab29 functions as an upstream GTPase of LRRK2 recruiting LRRK2 to the TGN resulting in an increase in LRRK2 kinase activity. LRRK2Rab29 interaction also seems required for constitutive phosphorylation of serine residues inbetween the ANK and LRR repeats. Kinases and GTPases, and their corresponding phosphorylation sites and interactors are colour coded using light yellow, blue and lilac. 
Figure 3

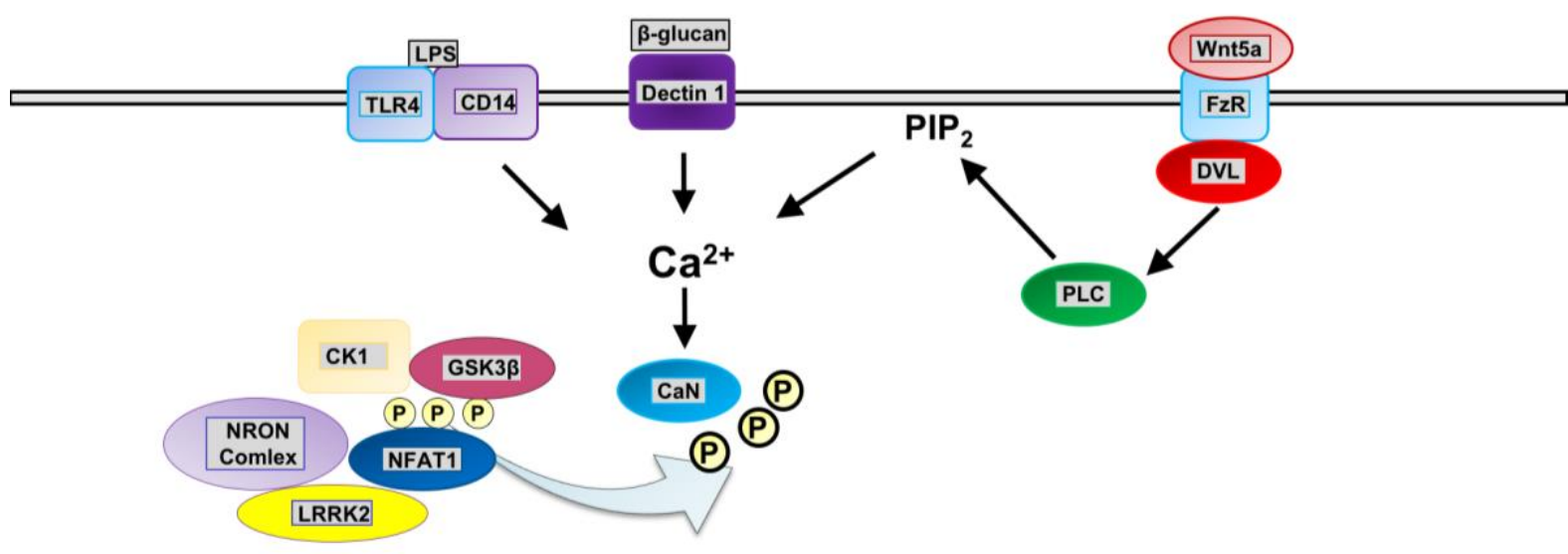

NFAT1

Transcription

\section{NFAT1}

Fig 3 LRRK2-mediated NFAT signalling in innate myeloid cells. LRRK2 knockout was shown to increase NFAT signalling in a model of Crohn's disease. Here, LRRK2 functions as a scaffold as part of the NRON complex facilitating NFAT phosphorylation by CK1, GSK3ß and DYRK1A. Upstream signalling, possibly through TLR4, Dectin and/or Wnt/Ca ${ }^{2+}$ signalling, leads to an increase in intracellular $\mathrm{Ca}^{2+}$, activation of the calcineurin phosphatase, dephosphorylation and nuclear localisation of NFAT, allowing NFAT downstream gene transcription. 
Figure 4

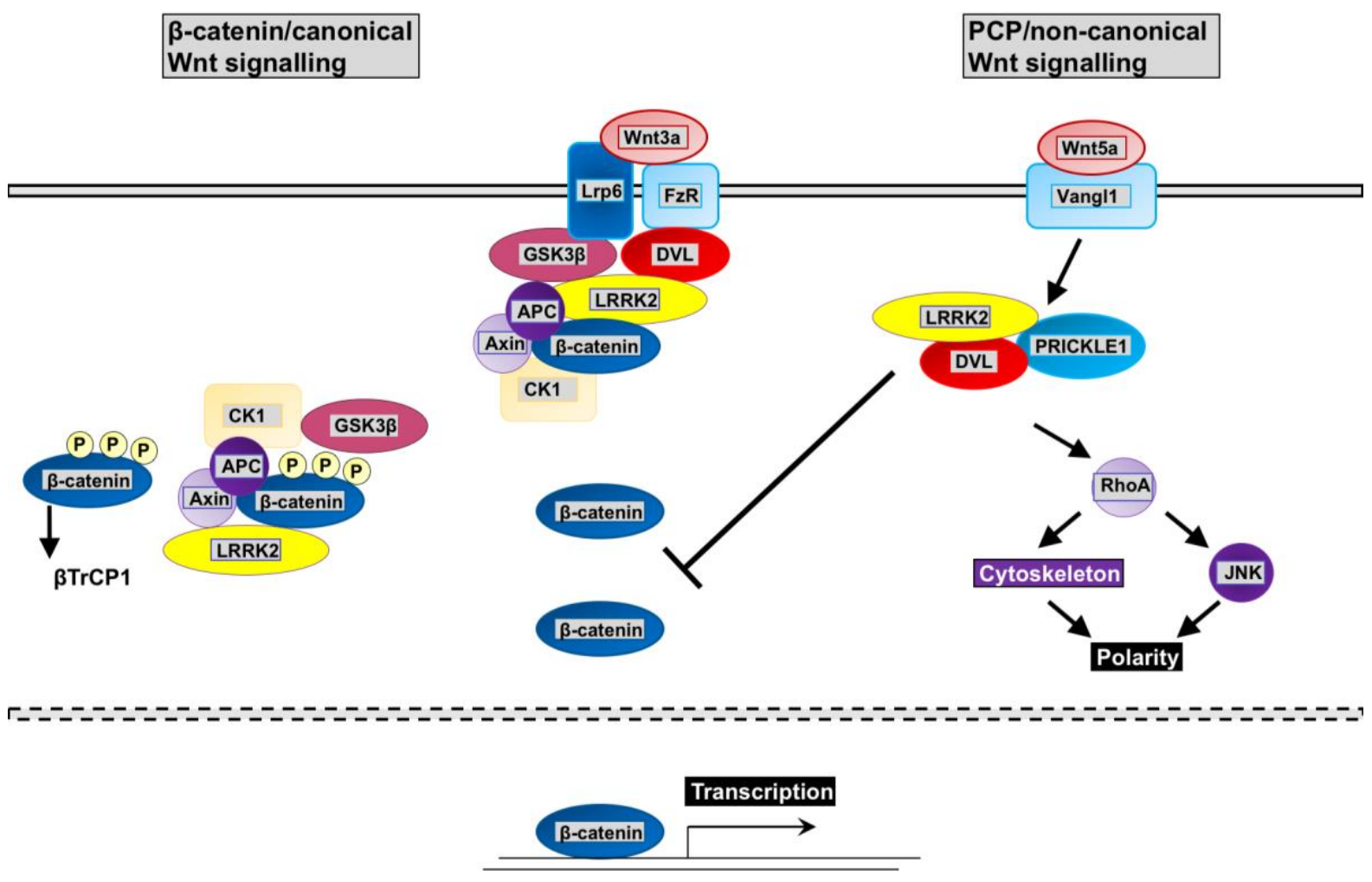

Fig 4 LRRK2-mediated Wnt signalling. LRRK2 was found to interact with several components of the canonical ß-catenin mediated (left) and non-canonical PCP (right) Wnt pathways. These two pathways are known to act mostly antagonistically. LRRK2 effects on the signalling pathways is highly dependent on the activation state of the pathways as well as interacting proteins. As part of the ß-catenin destruction complex LRRK2 interacts with GSK3ß, axin and ß-catenin allowing ß-catenin phosphorylation by CK1 and GSK3ß. This leads to the destruction of $\beta$-catenin in a proteasomal pathway. This suppressor function is further increased by LRRK2 PD mutations in line with a gain of LRRK2 function. The canonical Wnt signalling pathway is activated upon binding of canonical Wnt ligands such as Wnt3a to Frizzled receptors leading to the recruitment of the LRRK2 interacting DVL proteins and other components of the ß-catenin destruction complex to the cell membrane. Here LRRK2 interacts in addition with the Lrp6 canonical Wnt co-receptor and DVL proteins interact with Frizzled receptors. Under activating conditions LRRK2 functions as a canonical Wnt signalling enhancer. However, when LRRK2 is bound to the PCP pathway component PRICKLE1 it decreases canonical Wnt signalling and increases PCP signalling activity. 